\title{
Osteopontin expression and adventitial angiogenesis induced by local vascular endothelial growth factor 165 reduces experimental aortic calcification
}

\author{
Ralf G. Seipelt, MD ${ }^{a, b}$ \\ Carl L. Backer, MDa \\ Constantine Mavroudis, MD ${ }^{\mathrm{a}}$ \\ Veronica Stellmach, $\mathrm{PhD}^{\mathrm{c}}$ \\ Mona Cornwell, HAT $^{\mathrm{C}}$ \\ Ingrid M. Seipelt, MD ${ }^{c}$ \\ Friedrich A. Schoendube, $M D^{\text {b }}$ \\ Susan E. Crawford, MD ${ }^{\mathrm{C}}$
}

\footnotetext{
From the Department of Surgery, Division of Pediatric Cardiovascular Thoracic Surgery, Children's Memorial Hospital, Northwestern University Feinberg School of Medicine, Chicago, Ill, ${ }^{\text {a }}$ Department of Thoracic and Cardiovascular Surgery, Georg August University, Göttingen, Germany, ${ }^{\mathrm{b}}$ and Department of Pathology, Northwestern University Feinberg School of Medicine, Chicago, Ill. ${ }^{\mathrm{c}}$

This work was supported in part by $\mathrm{Na}$ tional Institutes of Health grant CA64329 and the Children's Heart Foundation.

Received for publication May 14, 2004; revisions received June 21, 2004; accepted for publication June 28, 2004

Address for reprints: Ralf G. Seipelt, MD, Department of Thoracic and Cardiovascular Surgery, Georg August University, University Hospital, Robert-Koch Str 40, 37075 Göttingen, Germany (E-mail: rseipelt@med. uni-goettingen.de).

J Thorac Cardiovasc Surg 2005;129:773-81 $0022-5223 / \$ 30.00$

Copyright (C) 2005 by The American Association for Thoracic Surgery

doi:10.1016/j.jtcvs.2004.06.039
}

\begin{abstract}
Background: Vascular calcification is a common pathologic and precisely regulated process involving bone-associated proteins such as osteopontin. In this study, we investigated mechanisms by which recombinant human vascular endothelial growth factor 165 protects the arterial wall from severe vascular remodeling, including calcification, a newly discovered biologic action of vascular endothelial growth factor.
\end{abstract}

Methods: In a rabbit model of thoracic aortic end-to-end anastomosis that simulates cardiovascular intervention, recombinant human vascular endothelial growth factor 165 at a dose of $0.75 \mu \mathrm{g}(\mathrm{n}=19)$ or albumin (control; $\mathrm{n}=19)$ was delivered intraluminally and on the serosal surface. Animals were killed, and aortic tissue was evaluated by Western blotting, immunohistochemistry, and immunofluorescence at 4,8 , and 24 hours; 1 week; and 1 month after surgery.

Results: All controls revealed extensive aortic medial calcification at 1 month, whereas calcification was significantly reduced or absent with vascular endothelial growth factor treatment. Compared with controls, vascular endothelial growth factor treatment resulted in an earlier infiltration of macrophages in the vessel media (at 8 hours: $5.7 \pm 2.3$ macrophages per high-power field in control vs $32.1 \pm 7.5$ in vascular endothelial growth factor-treated aortas; $P<.001$ ), whereas controls showed an increase in macrophages starting at 1 week $(24.1 \pm 6.9$ vs $4.3 \pm 1.8 ; P<.001)$. Osteopontin expression was transiently increased and detected in macrophages and endothelial cells in vascular endothelial growth factor-treated vessels, and adventitial microvascular density was significantly increased by 1 week $(9.5 \pm 0.43$ vs $25.0 \pm 1.3 ; P<.001)$.

Conclusions: Our data suggest that exogenous vascular endothelial growth factor is capable of increasing adventitial angiogenesis and shifting macrophage infiltration and osteopontin expression in the media to an earlier time point, thereby promoting prompt repair and diminishing vascular remodeling and calcification after acute vascular injury.

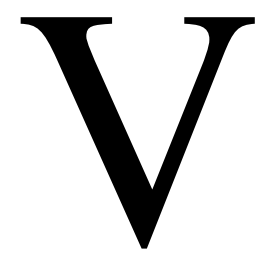

ascular remodeling, defined as any enduring change in the size or composition of a blood vessel, is a common disease affecting the cardiovascular system (with or without arterial calcification) in both children and adults. It is associated with numerous adverse events, including myocardial infarction, stroke, and hypertension. ${ }^{1-3}$ Calcification frequently accompanies vascular remodeling even when the disease is in its early stages of development. ${ }^{2}$ The mechanisms 
underlying vascular calcification are unclear, and therapeutic interventions to halt this disease process are limited. We recently described severe vascular remodeling with fibrosis, calcification, and chondroid metaplasia in a new model of thoracic aortic end-to-end anastomosis in rabbits. ${ }^{4}$ Locally applied recombinant human vascular endothelial growth factor (rhVEGF) was capable of reducing dystrophic medial calcification in this model. However, the mechanisms responsible for this new biological action of VEGF are unknown.

Earlier reports regarded vascular calcification as a passive degenerative process, but there is now strong evidence suggesting that arterial calcification is an actively regulated process with striking similarities to normal mineralization of bone and cartilage. ${ }^{5,6}$ Similar to normal bone, several bone-associated proteins have been found in vascular calcification, ${ }^{5,7-9}$ including osteopontin (OPN), a multifunctional arginine/glycine/aspartic acid phosphoprotein. ${ }^{10} \mathrm{OPN}$ has been observed at sites of calcification in human atherosclerotic plaques and in calcified aortic valves, ${ }^{7,9}$ suggesting that OPN may play a regulatory role in pathologic calcification. In atherosclerosis and calcific valves, macrophages seemed to be the major source of OPN, although both endothelial cells and vascular smooth muscle cells (VSMCs) are known to synthesize OPN., ${ }^{7,9,11}$ When exogenous OPN was added to a model of VSMC calcification, it was associated with hydroxyapatite crystals, and calcification was inhibited. ${ }^{12}$ Several stimuli regulate the expression of OPN, including inflammatory mediators and hypoxia. ${ }^{13}$ Because hypoxia regulates the expression of the angiogenic inducer VEGF, ${ }^{14}$ a mediator involved in the recruitment and activation of monocyte-macrophages, ${ }^{15,16}$ we postulated that VEGF may, directly or indirectly, regulate OPN expression in the arterial wall in the setting of acute vascular injury and, thus, alter vascular healing.

\section{Methods}

\section{Study Protocol}

A total of 38 male New Zealand White rabbits (9 weeks old; weight, $2.2-2.8 \mathrm{~kg}$ ) underwent aortic end-to-end anastomosis as previously described. ${ }^{4}$ Briefly, the thoracic aorta was dissected free just below the left subclavian artery, and after proximal and distal crossclamping, a 10-mm ring of thoracic aorta was resected. The end-to-end anastomosis was performed with running 7-0 polypropylene suture (Ethicon, Somerville, NJ). The distance between the crossclamps after resection was approximately $14 \mathrm{~mm}$. Treatment existed of applying either the $\operatorname{rhVEGF}_{165}(5 \mu \mathrm{g} / \mathrm{mL}$; R\&D Systems, Minneapolis, Minn) or the vehicle phosphatebuffered saline $/ 0.1 \%$ albumin as control to the intraluminal surface while the anastomosis was performed (10 minutes' exposure time) and after crossclamp release to the serosal surface $(1 \mathrm{~mm}$ beyond the crossclamp site, 10 minutes' exposure time) by using a $1-\mathrm{mL}$ syringe with a 27-gauge needle (Sherwood Medical, St Louis, Mo). A total volume of $0.15 \mathrm{~mL}$ of VEGF was split between the intraluminal surface and the adventitial surface and applied by dripping the solution on the surfaces. At no time did the needle make contact with the vessel wall. rhVEGF has been used in several animal models. ${ }^{17,18}$ The rabbits were allowed to recuperate and maintained under standardized conditions with a regular day/ night cycle (12/12 hours), free access to water, and standard rabbit diets (Prolab Hi-Fiber 5P25; PMI Nutrition International, St Louis, Mo). All studies were performed with approval of the Animal Care and Use Committee of Children's Memorial Institute of Education and Research and in accordance with the Guide for the Care and Use of Laboratory Animals (National Institutes of Health publication No. 85-23, revised 1996).

\section{Tissue Harvest and Histology}

At 4 hours $(n=4), 8$ hours $(n=8), 24$ hours $(n=8), 1$ week $(n=$ $10)$, and 1 month $(n=8)$, equal numbers of control and VEGF-treated rabbits were killed with intravenous pentobarbital $(150 \mathrm{mg} / \mathrm{kg})$. The heart, aortic arch, and thoracic aorta were immediately excised and gently flushed with saline. For histologic examination, the proximal segment of the anastomotic area $(9 \mathrm{~mm})$ was divided in 3 equal sections: the anastomosis itself, the portion including the proximal crossclamp, and the aortic wall between the anastomosis and the crossclamp. Parallel sections from each group were assessed, and a mean score was calculated for each aorta by using values obtained from all 3 segments. The tissue did not require decalcification. The tissue was fixed in $10 \%$ formalin overnight and embedded in paraffin, and $4-\mu \mathrm{m}$ sections were placed on a glass slide and stained with hematoxylin \& eosin, elastica van Gieson, and Von Kossa. The degree of fibrosis, calcification, and chondrocyte metaplasia was graded 1 to $4(1=$ none; $4=$ severe $)$ by a pathologist (S.E.C.) blinded to treatment regimen, as previously described. ${ }^{4}$ The aorta adjacent to the anastomotic suture line up to and including the distal crossclamp area was snap-frozen in liquid nitrogen and stored at $-80^{\circ} \mathrm{C}$.

\section{Immunohistochemistry}

Histologic sections of rabbit aortas were immunostained with antibodies directed against OPN (1B20; Assay Designs, Inc, Ann Arbor, Mich), macrophages (HAM 56; DAKO), von Willebrand factor-related antigen (DAKO, Carpinteria, Calif), or cartilage oligomeric matrix protein (Kamiya Biomedical Co, Seattle, Wash). Sections were incubated with an avidin-biotin peroxidaseconjugated secondary antibody and counterstained with hematoxylin or incubated with a fluorescence-conjugated (fluorescein isothiocyanate and phycoerythrin) secondary antibody. Control tissues were run in parallel to verify the specificity of the immunoreactivity where cell populations were known to be positive as well as negative (OPN/ kidney, macrophage/lymph node, endothelial cell/uninjured aorta, chondrocyte/trachea, and calcium/bone marrow). Negative controls were performed by omitting the primary antibody. Quantification of macrophages was performed at the different time points by counting 5 random high-power fields (hpf) per aorta by 2 independent observers blinded to the treatment regimen.

\section{Western Blot Analysis}

Frozen aortas were homogenized in radioimmunoprecipitation (RIPA) buffer. Protein concentration was determined with bicinchoninic acid protein assay reagent (Bio-Rad, Hercules, Calif). Equal protein ( $25 \mu \mathrm{g}$ per lane) was loaded into the wells of a $10 \%$ 

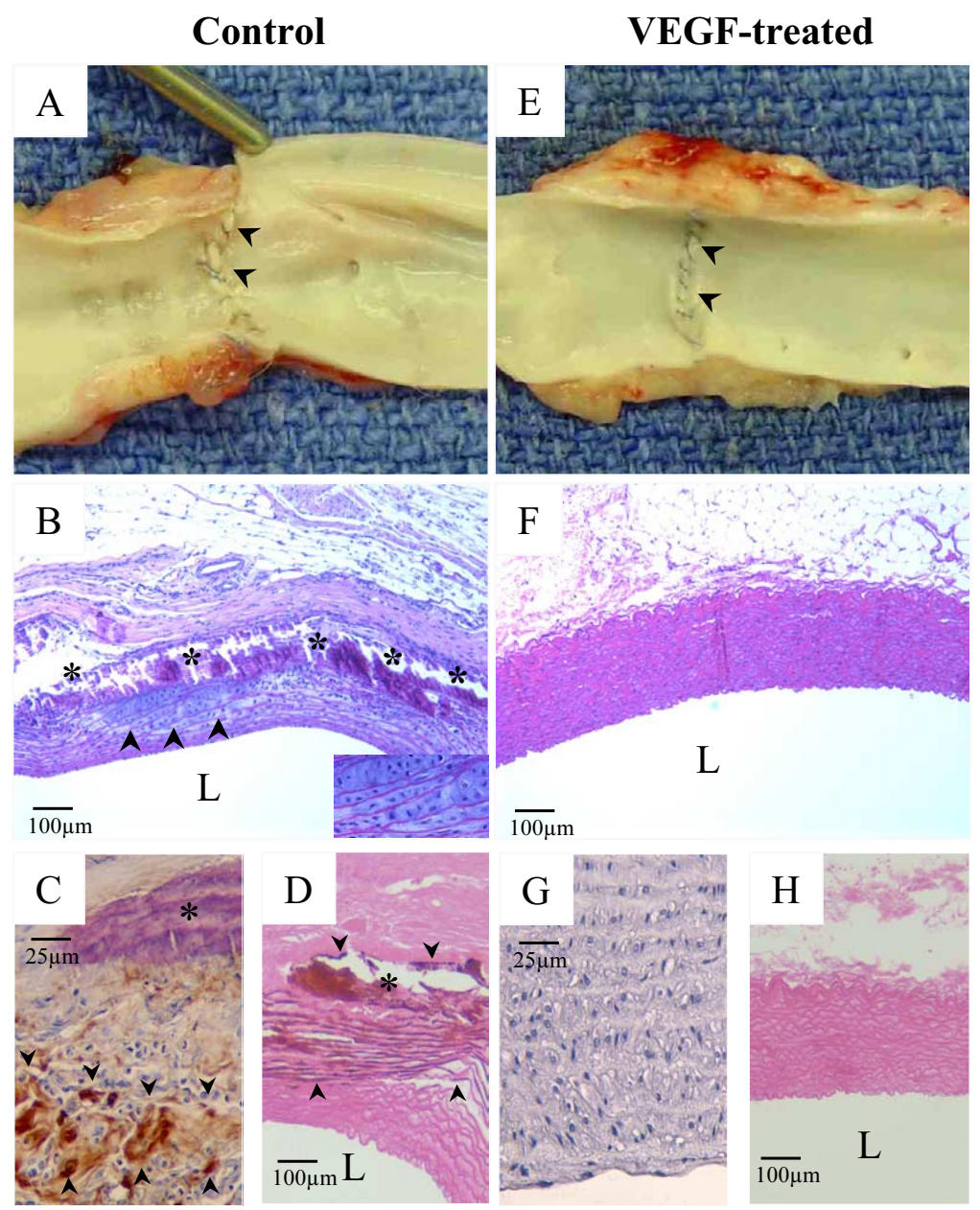

Figure 1. Local rhVEGF enhanced healing after aortic end-to-end anastomosis. Aortas were harvested 1 month after surgery. A and E, Gross evaluation; arrowheads indicate the suture line. B, Microscopic section of controls revealed a band of calcification (asterisks), and arrowheads and inset indicate cartilaginous tissue; VEGF-treated aortas had minimal pathology (F). C and G, Cartilage oligomeric matrix protein immunostaining revealed strong expression in the extracellular matrix of chondrocytes (arrowheads) adjacent to calcification (asterisk) in control aortas (C), whereas VEGF-treated aortas showed minimal staining in the media (G). D and H, Von Kossa-stained aorta; arrowheads indicate calcification. $L$, Lumen.

sodium dodecyl sulfate-polyacrylamide gel, subjected to electrophoresis under reducing conditions, and blotted onto Hybond-C nitrocellulose membranes (Amersham, Arlington Heights, Ill). After blocking with $5 \%$ nonfat dry milk/Tris-buffered saline $(\mathrm{pH}$ $8.0) / 1 \%$ Tween-20, the membrane was subsequently incubated with primary anti-OPN antibody (1:50; Assay Designs) and peroxidase-conjugated secondary anti-mouse immunoglobulin G (1: 1000; Santa Cruz Biotechnology, Santa Cruz, Calif). Signals were developed with Luminol reagent for Western blotting (Santa Cruz). Identically loaded Coomassie-stained gels confirmed equal protein loading. Molecular size standards (Bio-Rad) and recombinant OPN (30 ng per lane) were included as controls. Band intensities were quantified by densitometry with equalization to Coomassie-stained gels.
TABLE 1. Histologic characteristics of the arterial wall at 1 month

\begin{tabular}{lccc}
\hline Histologic feature & $\begin{array}{c}\text { Control } \\
\text { (mean } \pm \text { SEM) }\end{array}$ & $\begin{array}{c}\text { VEGF treated } \\
\text { (mean } \pm \text { SEM) }\end{array}$ & $\boldsymbol{P}$ value \\
\hline Fibrosis & $3.1 \pm 0.5$ & $1.5 \pm 0$ & .007 \\
Calcification & $3.6 \pm 0.5$ & $1.7 \pm 0.6$ & .004 \\
Chondroid metaplasia & $3.5 \pm 0.6$ & $1.7 \pm 0.6$ & .004 \\
\hline
\end{tabular}

VEGF, Vascular endothelial growth factor.

Determination of Microvascular Density

A pathologist and another observer, both blinded to treatment regimen, evaluated the aortic specimens on an independent basis. 


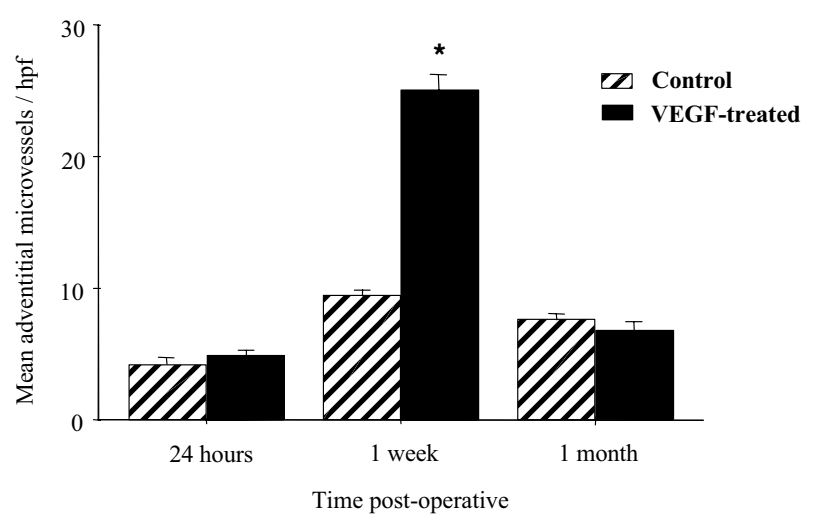

Figure 2. Local rhVEGF significantly increased adventitial angiogenesis by 1 week. ${ }^{*} P<.001$ compared with the control group. Results are mean \pm SEM.

The number of endothelial cell-lined vessels identified by antivon Willebrand factor staining in the adventitia was counted in 5 random hpf per histologic section. Values are expressed as mean \pm SEM. Data were analyzed by using the unpaired 2-tailed Student $t$ test and analysis of variance.

\section{Results}

\section{Effect of VEGF on Vascular Remodeling}

Gross examination of the control aortic specimens revealed marked changes at the anastomotic suture site and the adjacent aortic wall near the former crossclamp area, with more extensive plaques by 1 month (Figure $1, A$ ). In contrast, the suture line and the adjacent aorta of VEGF-treated specimens remained linear, without gross evidence of calcification (Figure 1,E). At earlier time points, control and VEGF-treated aortas appeared grossly similar. However, marked differences between the control and VEGF-treated groups were observed histologically as early as 24 hours after surgery. Control aortas at 1 month after surgery confirmed the gross findings, and cross sections from the suture site and the adjacent tissue revealed a bandlike region of dystrophic calcification in the media in all specimens (Figure $1, B)$. The calcium deposition, demonstrated by Von Kossa staining (Figure $1, D$ ) was so severe that it disrupted the integrity of the arterial wall, including the elastica. As observed previously, islands of mature-appearing cartilage (chondroid metaplasia; inset, Figure 1, B) identified by anti-cartilage oligomeric matrix protein staining (Figure 1, $C)$ were often in direct continuity with the pathologic calcification. ${ }^{4}$ In contrast, VEGF-treated aortas at 1 month revealed mild focal calcification with only a small island of chondrocytes at the anastomotic site, whereas tissue adjacent to this site showed no dystrophic calcification or chondroid metaplasia (Figure 1, F-H). The endothelial cell layers remained intact, and there was only mild fibrosis in the adventitia, with occasional extension into the media. When specimens harvested at 1 month after surgery from both groups were graded for degree of fibrosis, calcification, and chondroid metaplasia, the VEGF-treated aortas demonstrated significantly less disease (Table 1).

\section{Adventitial Angiogenesis}

In control specimens, the adventitia of the anastomotic area revealed acute inflammatory infiltrates as early as 8 hours after surgery, and the inflammatory process increased by 1 week. In the same group, adventitial neovascularization was limited to the foci of inflammation by 24 hours, with a mean adventitial microvascular density (MVD) of $4.2 \pm 0.6$ celllined vessel per hpf. The level of angiogenesis increased twofold by 1 week (mean MVD, $9.5 \pm 0.4$ cell-lined vessel per hpf) and remained increased at 1 month $(7.7 \pm 0.4$ cell-lined vessel per hpf). The VEGF-treated aortas showed only focal adventitial inflammation; however, several aggregates of capillaries were observed in the adventitia even in noninflamed regions within 24 hours $(5.0 \pm 0.5$ cell-lined vessel per hpf). By 1 week, the inflammatory infiltrates remained focal and were predominantly mononuclear cells. The adventitia showed a striking fivefold increase in MVD ( $25.0 \pm 1.3$ cell-lined vessel per hpf), significantly above the mean MVD of the 1-week control group (Figure 2; $P<$ $.001)$. However, the heightened angiogenesis was only temporary in the VEGF-treated group as the adventitial MVD decreased to $6.9 \pm 0.7$ cell-lined vessel per hpf by 1 month.

\section{Macrophage Infiltration}

Because VEGF is known to recruit macrophages in other circumstances, ${ }^{15,16}$ we determined whether there was an alteration in the macrophage population in the aortic media of the VEGF-treated and control groups. The pattern of macrophage infiltration in the aortic walls was significantly different between groups. When macrophages were counted in aortas harvested at 4,8 , and at 24 hours, the control aortas revealed only rare macrophages in the media (Figure 3, A and $B$ ), whereas the VEGF-treated group showed an early infiltration of macrophages in the media by 4 and 8 hours (mean number of macrophages per hpf: $5.7 \pm 0.4$ in control vs $32.1 \pm 1.8$ in VEGF-treated aortas; $P<.001$; Figure 3 , $D$ and $G$ ), and the macrophages persisted at 24 hours ( $4.4 \pm$ 0.3 in control versus $17.4 \pm 1.3$ in VEGF-treated aortas; $P<.001$, Figure 3, $E$ and $G$ ). At 1 week and beyond, the media of control aortas began to show an increase in macrophages, whereas the inflammatory cell infiltrate in the VEGF-treated group seemed to resolve $(24.1 \pm 1.8$ in control vs $4.3 \pm 0.2$ in VEGF-treated aortas; $P<.001$; Figure $3, G$ ). At 1 month, the aortic media of the control animals continued to demonstrate more inflammatory cells (Figure 3,C), unlike the rare macrophages noted in the VEGF-treated aortas (Figure 3, F). 


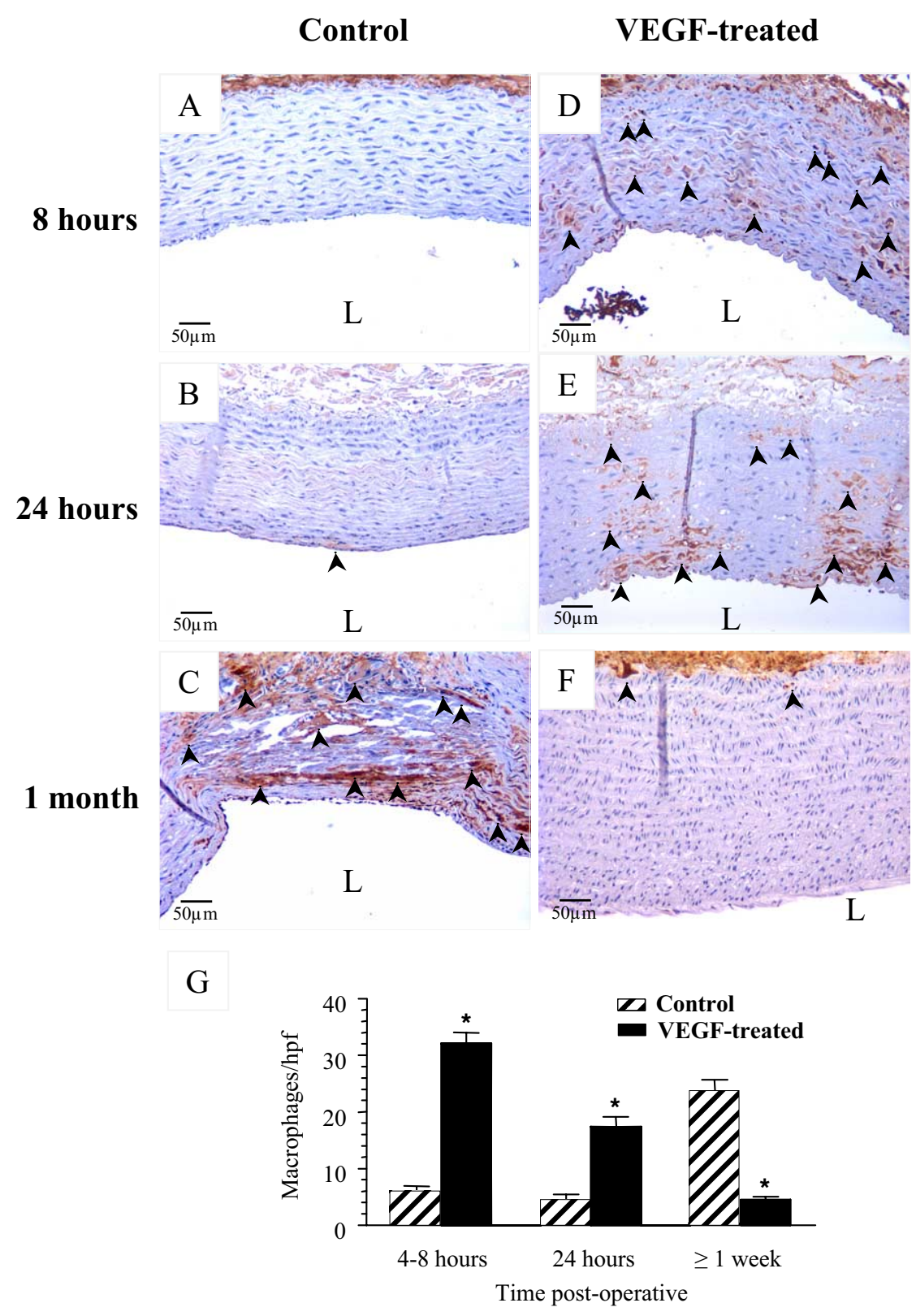

Figure 3. Injured control aortas (A-C) and VEGF-treated aortas (D-F) were examined for medial macrophage infiltration by immunohistochemistry at different time points. Arrowheads indicate macrophages. L, Lumen. G, Macrophages per high power field (hpf); results are mean \pm SEM. ${ }^{*} P<.001$ compared with the control group.

\section{OPN Expression}

OPN has been implicated in the pathogenesis of calcification $^{5,7-9,12,19}$ and has been shown to be induced by VEGF in vitro. ${ }^{20}$ To determine whether alteration in OPN expression was a target of topical VEGF treatment, we investigated the time course of expression of OPN protein by Western blotting. At time 0 , Western blotting revealed a low level of OPN in the aorta (Figure 4, A). At 8 hours after surgery, VEGF-treated aortas showed a strong band for OPN, with a 7.5-fold increase compared with time 0 . However, by 24 hours, OPN expression had diminished, and by 1 month, OPN expression returned to time 0 levels. In contrast, OPN expression of the control aortas peaked much later (7.7-fold increase) by 1 week, and its expression remained increased at 1 month (3.5-fold; $P<.05$ vs VEGF-treated aortas; Figure $4, A$ and $B$ ). Immunostaining was performed to differentiate the temporal OPN changes in the aortic adventitia and media. At time 0, mild OPN expression was de- 

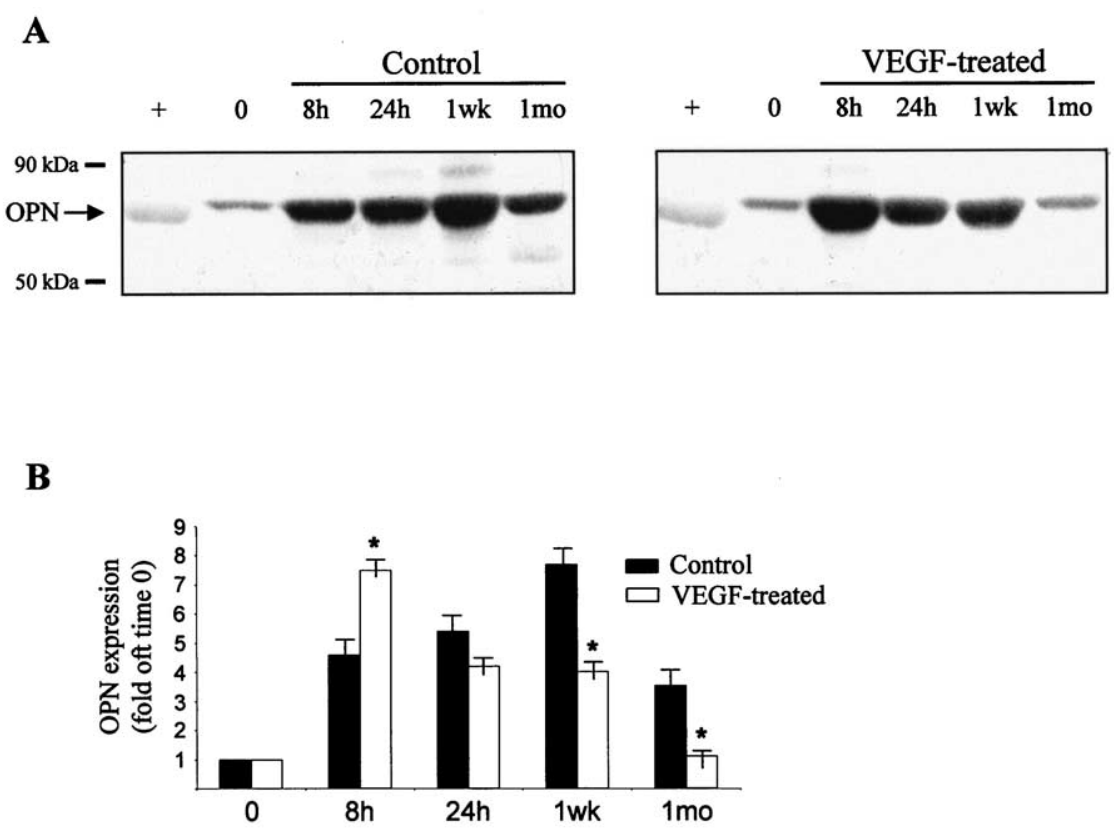

Figure 4. Representative Western blots showing an earlier peak in OPN protein level in VEGF-treated aorta (right panel, A) when compared with controls (left panel, A). +, Recombinant OPN (positive control). B, Corresponding densitometric analysis. Data are mean $\pm S E M ; n=3$ animals per time point and group. ${ }^{*} P<.05$ compared with the control group.

tected in the adventitia only, and this staining increased in both groups at 24 hours after surgery (data not shown). Similar to the pattern observed by Western analysis, adventitial OPN expression remained increased in control aortas at 1 month, whereas it returned almost to time 0 levels with VEGF treatment. In the VEGF-treated group, the time course of OPN expression in the media paralleled that of macrophage infiltration. In this group, there was a rapid increase in OPN, with strong staining detected in the luminal endothelial cells and in the immediate subjacent tissue by 4 hours after injury (Figure $5, D$ ), whereas the control group showed mild staining in a rare endothelial cell (Figure 5, A). By 24 hours, the VEGF-treated group had OPN localized to the entire media (Figure $5, E$ ) in infiltrating macrophages (inset, Figure 5, E). Immunofluorescence double staining revealed that $40 \%$ of macrophages in the vessel media expressed OPN (data not shown). This was in contrast to the weak endothelial staining and faint medial staining observed in the control group (Figure 5, B). The difference in OPN staining between groups was most apparent at 1 week. At this time point, in the VEGF-treated group, OPN staining was retained by the endothelial cells, and the media was predominantly negative, with only focal positivity at the interface between the media and adventitial layers of the aorta (Figure 5, F). In contrast, the control specimens showed intense staining for OPN in the media and adventitia and only focal positivity in endothelial cells (Figure 5,
$C)$. This high level of OPN expression persisted at 1 month after surgery.

\section{Discussion}

The endothelial cell-specific mitogen VEGF has drawn widespread attention for its therapeutic value of stimulating angiogenesis in cardiovascular disease. ${ }^{21,22}$ However, depending on the physiologic context or experimental model used, rhVEGF or VEGF gene transfer can have beneficial, adverse, or no effects at all on the vessel wall. In a model of neointimal VSMC hyperplasia in which the endothelium is not initially damaged, VEGF gene therapy strikingly inhibited neointimal VSMC hyperplasia, ${ }^{23}$ whereas, when the endothelium is denuded in the balloon arterial injury model, local intravascular administration of VEGF accelerated reendothelialization, thus leading to marked reduction in intimal thickening. ${ }^{24}$ Another more controversial study showed that in apolipoprotein E-deficient mice, a single intraperitoneal dose of rhVEGF stimulated macrophage infiltration in the atherosclerotic plaque and actually enhanced its progression. ${ }^{17,25}$ We recently observed a new biological action of VEGF to trigger suppression of pathologic vascular calcification and chondroid metaplasia. ${ }^{4}$ Here we present several potential mechanisms underlying the ability of VEGF to protect the arterial wall from severe remodeling after aortic end-to-end anastomosis as performed to repair a coarctation of the aorta. One possible mechanism is related 


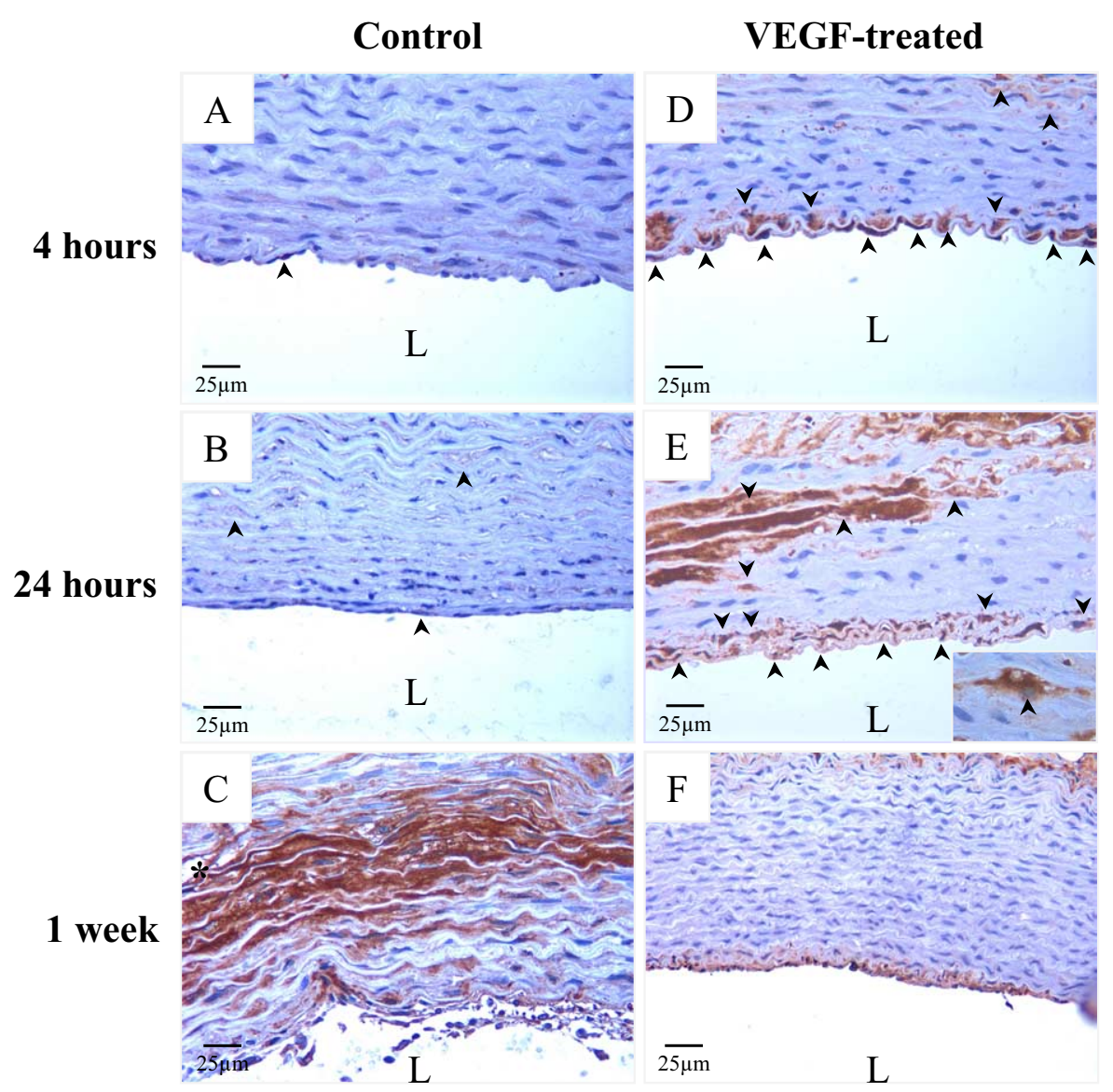

Figure 5. OPN immunostaining in control (A-C) and VEGF-treated (D-F) aortas at different times points. Arrowheads indicate OPN positivity. Inset in (E) demonstrates an OPN ${ }^{+}$macrophage. $L$, Lumen.

to the ability of VEGF to induce endothelial cell proliferation. ${ }^{14}$ VEGF-treated aortas demonstrated an increase in adventitial angiogenesis, as indicated by significantly higher MVD. The neovascularization was seemingly unrelated to the degree of inflammation and was transient. It is possible that this brief exposure to an angiogenic stimulus resulting in neovascularization could help to overcome acute hypoxia associated with the aortic end-to-end anastomosis. ${ }^{26}$

In addition to the activity of VEGF on the adventitia, our results point to 2 other possible mechanisms in another segment of the arterial wall, the media. The first observation involves earlier recruitment of macrophages, and the second is related to earlier expression of OPN at the site of injury (Figure 6). Previous studies have found that VEGF can act as a chemotactic factor for monocyte-macrophages through its receptor Flt-1 (VEGF receptor-1). ${ }^{15,16}$ We found that VEGF shifted the infiltration of macrophages in the arterial media to an earlier postinjury time point than in the control aortas. This earlier response may be important in wound healing, but it may also suppress calcific deposits, because the macrophages have been reported to clear the injured aortic wall of dead cell bodies that can then act as nucleating structures for calcification. ${ }^{27}$ Moreover, mononuclear cells might undergo differentiation toward osteoclast-like cells, as proposed by Doherty and colleagues, ${ }^{28}$ and thus restrict calcification.

OPN has been noted in areas of dystrophic calcification as atherosclerotic plaques and calcified aortic valves, ${ }^{7,9}$ and both in vitro ${ }^{12}$ and in vivo ${ }^{19}$ studies have suggested that the upregulation of OPN in areas of dystrophic calcification is an attempt to limit vascular calcification. OPN has also been found in lesions after arterial injury, thus suggesting that it plays a pathologic role in intimal hyperplasia. ${ }^{29}$ These apparently opposing actions of OPN on the injured arterial wall made it difficult to predict whether OPN would act as an inducer or inhibitor of vascular remodeling. Our data indicated that the time course of OPN expression after arterial injury may be crucial for determining its course of action. OPN was substantially upregulated in the VEGFtreated aortas, and, similar to the timing of the macrophages, OPN expression occurred much earlier than in the control aortas (Figure 6). Both macrophages and endothelial cells 


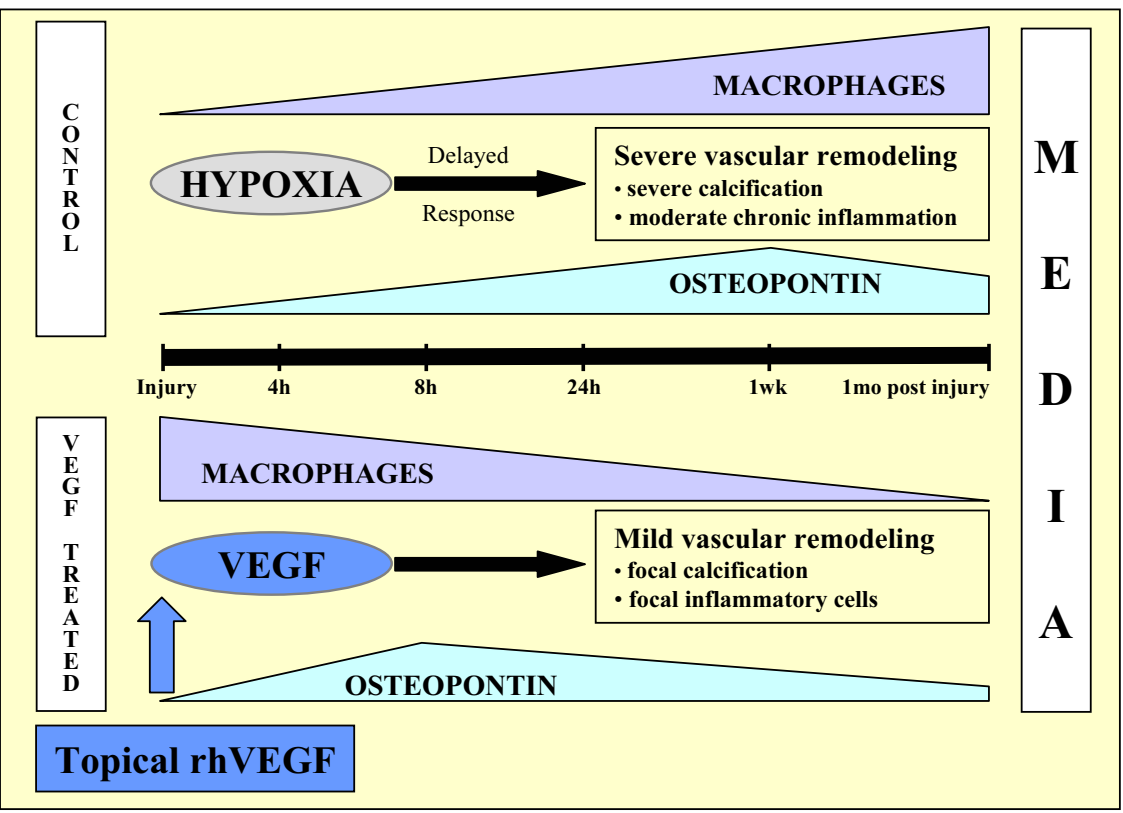

Figure 6. Proposed model of VEGF-induced protection of the arterial wall after hypoxic injury. Topical rhVEGF was able to shift macrophage infiltration and OPN expression to an earlier time point (bottom) and enhanced healing after aortic injury. A delayed response, as observed in control aortas (top), resulted in severe vascular remodeling with moderate chronic inflammation and persistent OPN expression in the vascular media.

seemed to be sources of OPN after injury. Control aortas had sustained expression of OPN and persistent macrophage infiltration. One or both of these factors could impair proper wound healing.

Our findings in this experimental model suggest that VEGF can protect vessels from pathologic remodeling by stimulating adventitial angiogenesis, recruiting macrophages, and upregulating vascular OPN levels in the early phase after arterial injury. Further studies are needed to determine whether OPN alone is sufficient to diminish pathologic vascular calcification. Moreover, these findings suggest that current protocols testing VEGF as an agent for therapeutic angiogenesis in ischemic tissue may have another unexpected therapeutic benefit: reduction of the calcific deposits that often complicate vascular stenoses.

\section{References}

1. Fuster V, Badimon L, Badimon JJ, Chesebro JH. The pathogenesis of coronary artery disease and the acute coronary syndromes. $N$ Engl J Med. 1992;326:242-50.

2. Wexler L, Brundage B, Crouse J, Detrano R, Fuster V, Maddahi J, et al. Coronary artery calcification: pathophysiology, epidemiology, imaging methods, and clinical implications. A statement for health professionals from the American Heart Association. Writing Group. Circulation. 1996;94:1175-92.

3. Mahoney LT, Burns TL, Stanford W, Thompson BH, Witt JD, Rost CA, et al. Coronary risk factors measured in childhood and young adult life are associated with coronary artery calcification in young adults: the Muscatine Study. J Am Coll Cardiol. 1996;27:277-84.
4. Seipelt RG, Backer CL, Mavroudis C, Stellmach V, Seipelt IM, Cornwell M, et al. Topical VEGF enhances healing of thoracic aortic anastomosis for coarctation in a rabbit model. Circulation. 2003;108: II150-4.

5. Canfield AE, Farrington C, Dziobon MD, Boot-Handford RP, Heagerty AM, Kumar SN, et al. The involvement of matrix glycoproteins in vascular calcification and fibrosis: an immunohistochemical study. J Pathol. 2002;196:228-34.

6. Bostrom K, Demer LL. Regulatory mechanisms in vascular calcification. Crit Rev Eukaryot Gene Expr. 2000;12:151-8.

7. O’Brien KD, Kuusisto J, Reichenbach DD, Ferguson M, Giachelli C, Alpers CE, et al. Osteopontin is expressed in human aortic valvular lesions. Circulation. 1995;92:2163-8.

8. Gadeau AP, Chaulet H, Daret D, Kockx M, Daniel-Lamaziere JM, Desgranges C. Time course of osteopontin, osteocalcin, and osteonectin accumulation and calcification after acute vessel wall injury. $J$ Histochem Cytochem. 2001;49:79-86.

9. Fitzpatrick LA, Severson A, Edwards WD, Ingram RT. Diffuse calcification in human coronary arteries. Association of osteopontin with atherosclerosis. J Clin Invest. 1994;94:1597-604.

10. Giachelli CM, Steitz S. Osteopontin: a versatile regulator of inflammation and biomineralization. Matrix Biol. 2000;19:615-22.

11. O'Brien ER, Garvin MR, Stewart DK, Hinohara T, Simpson JB, Schwartz $\mathrm{SM}$, et al. Osteopontin is synthesized by macrophage, smooth muscle, and endothelial cells in primary and restenotic human coronary atherosclerotic plaques. Arterioscler Thromb. 1994;14:1648-56.

12. Wada T, McKee MD, Steitz S, Giachelli CM. Calcification of vascular smooth muscle cell cultures: inhibition by osteopontin. Circ Res. 1999;84:166-78.

13. Denhardt DT, Guo X. Osteopontin: a protein with diverse functions. FASEB J. 1993;7:1475-82.

14. Ferrara N. Role of vascular endothelial growth factor in regulation of physiological angiogenesis. Am J Physiol Cell Physiol. 2001;280: 1358-66. 
15. Clauss M, Gerlach M, Gerlach H, Brett J, Wang F, Familletti PC, et al. Vascular permeability factor: a tumor-derived polypeptide that induces endothelial cells and monocyte procoagulant activity, and promotes monocyte migration. J Exp Med. 1990;172:1535-45.

16. Barleon B, Sozzani S, Zhou D, Weich HA, Mantovani A, Marme D Migration of human monocytes in response to vascular endothelial growth factor (VEGF) is mediated via the VEGF receptor flt-1. Blood. 1996;87:3336-43.

17. Celletti FL, Waugh JM, Amabile PG, Brendolan A, Hilfiker PR, Dake MD. Vascular endothelial growth factor enhances atherosclerotic plaque progression. Nat Med. 2001;7:425-429.

18. Dodge-Khatami A, Backer CL, Holinger LD, Mavroudis C, Cook KE, Crawford SE. Healing of a free tracheal autograft is enhanced by topical vascular endothelial growth factor in an experimental rabbit model. J Thorac Cardiovasc Surg. 2001;122:554-61.

19. Speer MY, McKee MD, Guldberg RE, Liaw L, Yang HY, Tung E, et al. Inactivation of the osteopontin gene enhances vascular calcification of matrix Gla protein-deficient mice: evidence for osteopontin as an inducible inhibitor of vascular calcification in vivo. J Exp Med. 2002; 196:1047-55.

20. Senger DR, Ledbetter SR, Claffey KP, Papadopoulos-Sergiou A, Peruzzi CA, Detmar M. Stimulation of endothelial cell migration by vascular permeability factor/vascular endothelial growth factor through cooperative mechanisms involving the alphavbeta3 integrin, osteopontin, and thrombin. Am J Pathol. 1996;149:293-305.
21. Ferrara N, Alitalo K. Clinical applications of angiogenic growth factors and their inhibitors. Nat Med. 1999;5:1359-64.

22. Rivard A, Isner JM. Angiogenesis and vasculogenesis in treatment of cardiovascular disease. Mol Med. 1998;4:429-40.

23. Laitinen M, Zachary I, Breier G, Pakkanen T, Hakkinen T, Luoma J, et al. VEGF gene transfer reduces intimal thickening via increased production of nitric oxide in carotid arteries. Hum Gene Ther. 1997; 8:1737-44.

24. Asahara T, Bauters C, Pastore C, Kearney M, Rossow S, Bunting S, et al. Local delivery of vascular endothelial growth factor accelerates reendothelialization and attenuates intimal hyperplasia in ballooninjured rat carotid artery. Circulation. 1995;91:2793-801.

25. Isner JM. Still more debate over VEGF. Nat Med. 2001;7:639-41.

26. Kivisaari J, Niinikoski J. Oxygen tensions in healing anastomosis of rabbit aorta. Surgery. 1975;78:165-75.

27. Proudfoot D, Skepper JN, Hegyi L, Bennett MR, Shanahan CM, Weissberg PL. Apoptosis regulates human vascular calcification in vitro: evidence for initiation of vascular calcification by apoptotic bodies. Circ Res. 2000;87:1055-62.

28. Doherty TM, Uzui H, Fitzpatrick LA, Tripathi PV, Dunstan CR, Asotra K, et al. Rationale for the role of osteoclast-like cells in arterial calcification. FASEB J. 2002;16:577-82.

29. Isoda K, Nishikawa K, Kamezawa Y, Yoshida M, Kusuhara M, Moroi $\mathrm{M}$, et al. Osteopontin plays an important role in the development of medial thickening and neointimal formation. Circ Res. 2002;91:77-82. 